\title{
Lifetime Maximization of Wireless Video Sensor Network Node by Dynamically Resizing Communication Buffer
}

\author{
Kang-Woo Choi ${ }^{1}$, Kang $\mathrm{Yi}^{2}$ and Chong Min Kyung ${ }^{3}$ \\ ${ }^{1}$ Samsung Electronics, Suwon, Korea \\ [e-mail: ckangw@gmail.com] \\ ${ }^{2}$ School of Computer Science and Electrical Engineering, Handong Global University,Pohang, Korea \\ [e-mail: yk@handong.edu] \\ ${ }^{3}$ School of Electrical Engineering, KAIST, Daejeon, Korea \\ [e-mail: kyung@kaist.ac.kr] \\ *Corresponding author: Kang Yi
}

Received April 13, 2017; revised September 24, 2017; accepted October 8, 2017; published October 31, 2017

\begin{abstract}
Reducing energy consumption in a wireless video sensor network (WVSN) is a crucial problem because of the high video data volume and severe energy constraints of battery-powered WVSN nodes. In this paper, we present an adaptive dynamic resizing approach for a SRAM communication buffer in a WVSN node in order to reduce the energy consumption and thereby, to maximize the lifetime of the WVSN nodes. To reduce the power consumption of the communication part, which is typically the most energy-consuming component in the WVSN nodes, the radio needs to remain turned off during the data buffer-filling period as well as idle period. As the radio ON/OFF transition incurs extra energy consumption, we need to reduce the ON/OFF transition frequency, which requires a large-sized buffer. However, a large-sized SRAM buffer results in more energy consumption because SRAM power consumption is proportional to the memory size. We can dynamically adjust any active buffer memory size by utilizing a power-gating technique to reflect the optimal control on the buffer size. This paper aims at finding the optimal buffer size, based on the trade-off between the respective energy consumption ratios of the communication buffer and the radio part, respectively. We derive a formula showing the relationship between control variables, including active buffer size and total energy consumption, to mathematically determine the optimal buffer size for any given conditions to minimize total energy consumption. Simulation results show that the overall energy reduction, using our approach, is up to $40.48 \%$ ( $26.96 \%$ on average) compared to the conventional wireless communication scheme. In addition, the lifetime of the WVSN node has been extended by $22.17 \%$ on average, compared to the existing approaches.
\end{abstract}

This work was supported by the Center for Integrated Smart Sensors funded by the Ministry of Science, ICT \& Future Planning as Global Frontier Project" (CISS-2011-0031863)

A preliminary version of this paper was presented at ICONI 2016, and was selected as an outstanding paper 
Keywords: Wireless Video Sensor Network, Communication Buffer Sizing, Lifetime Maximization of WVSN nodes, Minimizing Energy Consumption of Sensor Node

\section{Introduction}

Demands on wireless video sensor networks (WVSN) keep increasing, owing to the steady increase of societal demands due to security and safety issues. WVSN has a range of applications, including surveillance, environmental tracking, and the monitoring of natural disasters. Innovations in semiconductor manufacturing, system-on-chip design methodologies to integrate digital, analog, and RF circuits in a single silicon die [1] as well as, low-power embedded system design technologies, have enabled the development of small, inexpensive, and energy-efficient WVSN sensor node systems.

The WVSN sensor nodes need to be equipped with an event-detector, CMOS image sensor (CIS), video compressor, communication block, microcontroller, and a battery and/or energy harvester. One of the most challenging problems in WVSN design is to reduce energy consumption level because the nodes in a WVSN are battery-powered. There are many research works to reduce the energy consumption of sensor nodes and thereby maximize the lifetime of WVSN systems. In [2,3] , to prolong the lifetime of nodes, inactive parts of a node, except the event detector, are normally in power-down mode and only are woken up by the event detector.

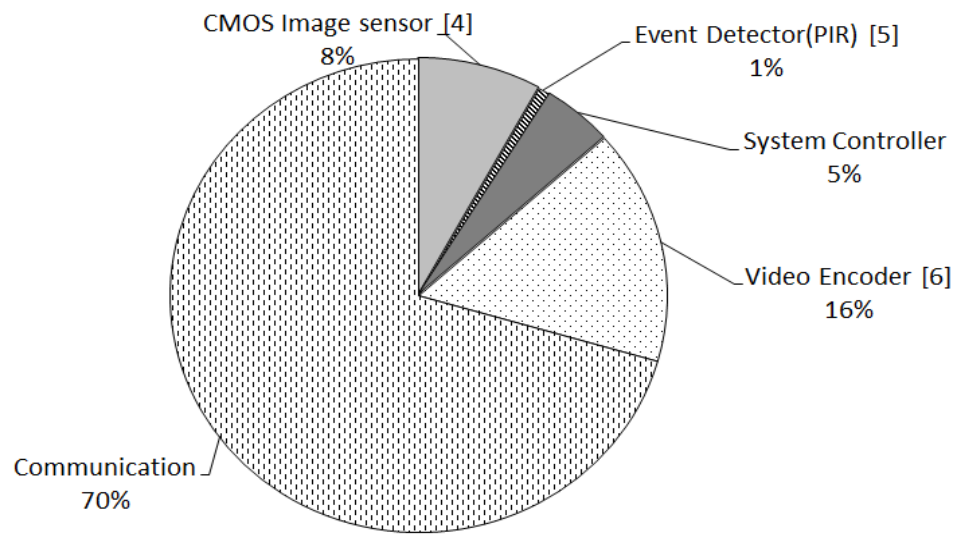

Fig. 1. The Power Consumption Ratio of each part of the WVSN node

Fig. 1 shows the power demand of each block of WVSN node with a wireless data link. It is shown that the wireless communication part has the highest energy consumption of the components. According to our WVSN node power analysis, the power consumption of the communication block, including the communication buffer, accounts for more than half of the system power consumption. 
Zainaldin et al. [4] proposed a technique to extract and transmit the region of interest (ROI) from the video data to fit the 802.15.4 data rate, but did not consider the power requirement amount for the ROI extraction. There are many research works attempting to solve the energy problems of WVSN systems. Schurgers et al. [5] proposed an energy efficient routing that selects energy efficient paths in a network along which to send traffic, thereby minimizing communication energy. To consume the least amount of power, devices are in sleep state as much as possible and only are awoken in the case of data transmissions, so the IEEE 802.11 group adopted the idea as a power saving mode (PSM) concept [6]. Enhua et al. [7] proposed the/a PSM-throttling method that minimizes energy consumption for bulky data communications. In [8], to minimize the activity of a node by adopting event-driven wake-up techniques, inactive parts, with the exception of the event detector, are normally in power-down states and are only activated by the event detector. However, no other research work/study has addressed the issue overall transceiver power consumption, including buffer memory power consumption, and the start-up overhead of the communication module.

Table 1 shows the 802.11g (Wi-Fi) radio performance and power consumption for transmission (Tx), receiving (Rx), and IDLE state in case of maximum throughput and practical throughput in congested environments. Table 1 also shows the start-up time/energy overhead of Wi-Fi radio from a SLEEP state to Tx state.

Table 1. Wi-Fi chip (CC3000) characteristics

\begin{tabular}{|c|c|c|c|}
\hline & $\begin{array}{c}\text { Power } \\
\text { Consumption of } \\
\text { receive / transmit / } \\
\text { idle (mW) }\end{array}$ & $\begin{array}{c}\text { Speed } \\
\text { max bps / } \\
\text { effective bps }\end{array}$ & $\begin{array}{c}\text { Start-up } \\
\text { time / energy }\end{array}$ \\
\hline \hline $802.11 \mathrm{~g}$ & $303.6 / 617.1 / 230$ & $\begin{array}{c}54 \mathrm{Mbps} / \\
16 \mathrm{Mbps}[16]\end{array}$ & $1.3 \mathrm{~s} / 299 \mathrm{~mJ}$ \\
\hline$(\mathrm{CC} 3000)$ & &
\end{tabular}

Note that Wi-Fi consumes a high amount of power, even in the IDLE state, and there are energy overheads for mode transition from a SLEEP to a Tx state (Start-up). To conserve Wi-Fi energy consumption we, therefore, need to turn off the Wi-Fi radio while the communication buffer is receiving data. The power consumption of the Wi-Fi radio, including the power amplifier, is bigger than that of the communication buffer by about 5 times when 2MB SRAM is used as the/a communication buffer, as will be shown later ${ }^{1}$. The radio needs to stay in the/its turned-off state as long as possible and the radio on/off transition frequency needs be as small as possible for energy saving purposes. Our finding is that the buffer size affects both the energy consumption of the buffer itself, as well as the energy consumption of the Wi-Fi radio. The effect of the buffer size to the energy consumption of Wi-Fi radio is opposite to the energy consumption of the communication buffer itself. Thus, the two energy consumption factors are complementary, which leads us to determine the buffer size to achieve optimal energy consumption levels.

\footnotetext{
${ }^{1}$ A: Wi-Fi Transmission energy consumption $=617.1 \mathrm{~mW}$ (Table 1), B: 2 MByte SRAM total power consumption $=118.72 \mathrm{~mW}$ (Fig. 2), $\mathrm{A} / \mathrm{B} \approx 5$
} 
Fig. 2 illustrates the energy consumption of the buffer and radio, as the buffer size is different in the case of PSM transmission, in which radio power is turned off in the/an idle state. In Fig. 2 (a), where the buffer size is $1 \mathrm{MB}, 60 \mathrm{~mW}$ is consumed by buffer memory. In Fig. 2 (b), where the buffer size is $2 \mathrm{MB}$, of which $119 \mathrm{~mW}$ is consumed by buffer memory. According to the data in Table 1, the start-up power of $230 \mathrm{~mW}$ is consumed in the radio from [Turn-on radio] to [Transmission-start], while full transmission power of $617 \mathrm{~mW}$ is consumed from [Transmission-start] to [Transmission-end].

Due to these power consumption features of the communication part, we discuss a Wi-Fi power management scheme, which includes buffer resizing for less energy consumption of the WVSN node. Due to these power consumption features of the communication part, we discuss a Wi-Fi power management scheme, which includes buffer resizing

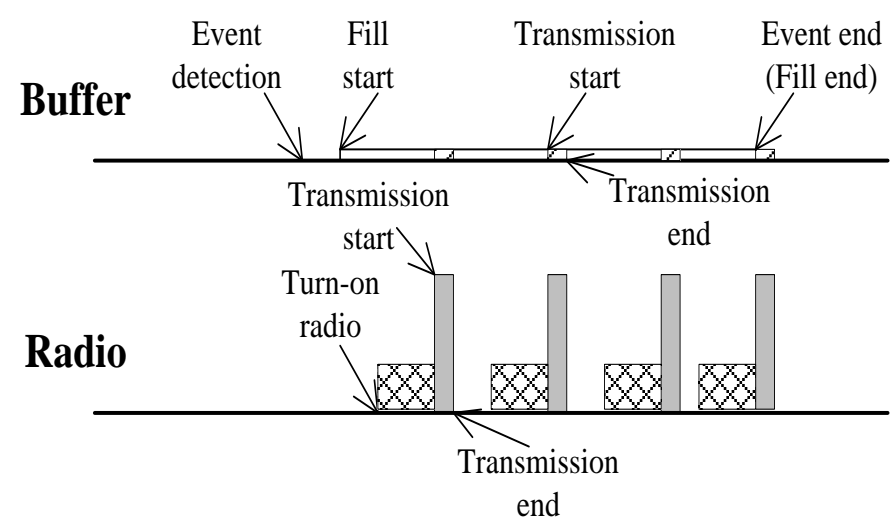

(a)

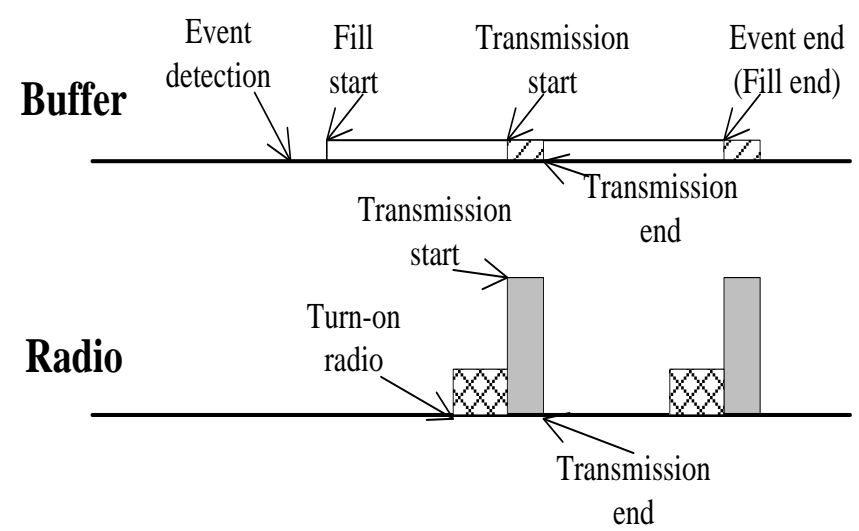

(b)

Fig. 2. Operation mode and energy consumption of the buffer and radio for two different buffer sizes:

(a) Buffer size is $1 M B$ and buffer power consumption is $60 \mathrm{~mW}$,

(b) Buffer size is $2 M B$ and buffer power consumption is $119 \mathrm{~mW}$

In this paper, we propose a WVSN communication system with optimal buffer size to address the communication energy minimization problem. Our contribution is two-fold. First, we suggest an optimal buffer size selection method that is based on a mathematical model. Second, we propose a run-time buffer resizing scheme that takes into account data size and channel conditions to minimize the overall energy consumption of WVSN data transmissions. 
At our knowledge, we are the first who applied the power gating technology to the communication buffer memory for physical size change in a run time. In addition, as far as we know we are the first who think changing the communication buffer size in a real time manner to save energy. According to the simulation results, the energy consumption of our approach achieved from $13.8 \%$ to $40.48 \%$ saving for ideal wireless communication channel condition, and from 12.01 to $29.79 \%$ saving for practical effective wireless communication channel condition, respectively, compared to the conventional approach that uses a fixed buffer size.

In Section 2, we present our WVSN overview and the motivation for our research. Section 3 presents our modeling and mathematical formulas derived to find the best solution. Section 4 shows the experimental results, and Section 5 concludes the paper.

\section{System Overview and Motivation}

\subsection{System Overview}

Our system consists of a passive infrared (PIR) sensor for event detection, CMOS image sensor (CIS), video encoder, system controller and a wireless transceiver, including a buffer for sending and receiving data, as shown in Fig. 3. Most of the time (more than 90\%) the system, except for the PIR sensor, resides in a/its sleep state until alerted by an event. When the PIR sensor detects an event, CIS (CMOS image sensor) captures it and collects the video in proper sampling rates. The video data is encoded by the H.264/AVC video encoder complete with a baseline profile, producing a moderate compression rate (about 0.6 bits per pixel). The encoded video data is stored temporarilly in the buffer for wireless transmission purpose.

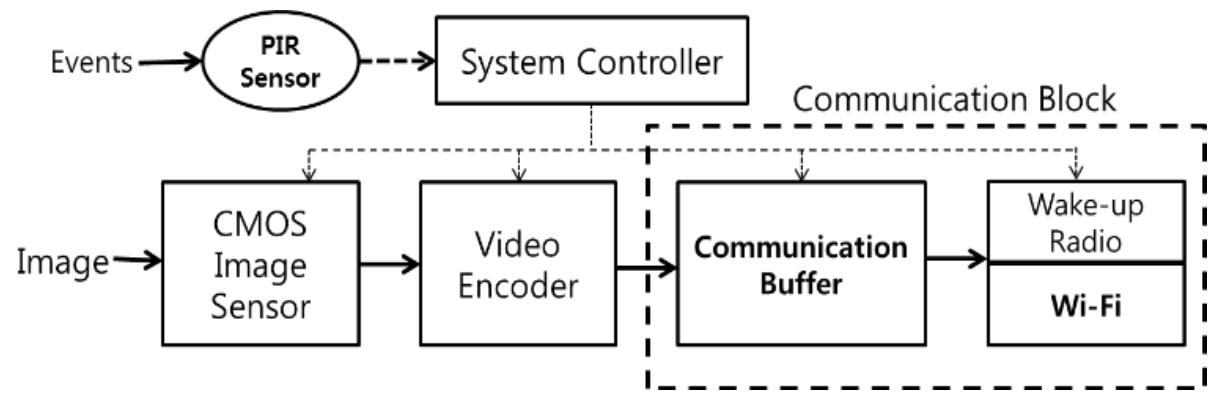

Fig. 3. Our WVSN node architecture

In our sensor node, the transceiver part consists of a wake-up radio and a Wi-Fi radio. The wake-up radio [9] is a special radio designated to wake up the main radio, the Wi-Fi radio, with minimal energy consumption. Fig 4 illustrates a typical process of the node when an event occurs. The PIR monitors events and wakes up the whole system when it detects any event. For every event, the controller orchestrates the system to record and transmit the event images to other node(s). 


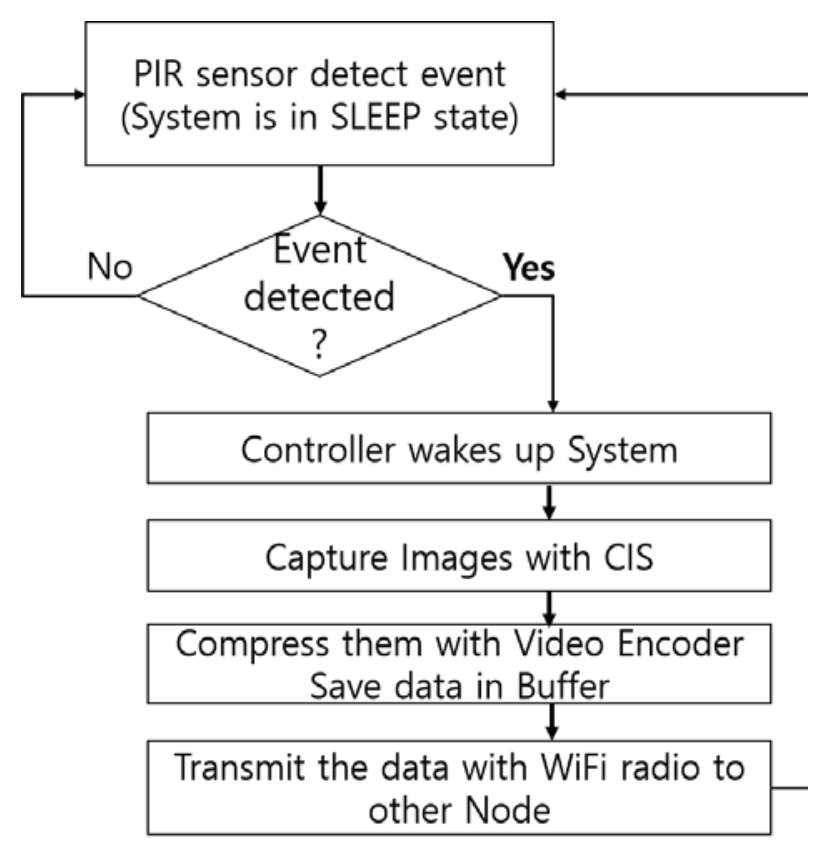

Fig. 4. The overall control flow for each of our WVSN node operations

\subsection{Wi-Fi Operation Modes and Communication Power Consumption}

There are five basic operation states in the transceiver, namely, SLEEP, IDLE, STANDBY, $\mathrm{Tx}$, and Rx. The transceiver is in a Tx/Rx state when sending or receiving data. When there is no network traffic, it is in an IDLE or SLEEP or STANDBY state, depending on the communication state. In its IDLE state, the communication buffer and radio are both active to maintain channel access data communications. In its SLEEP state, the communication buffer and radio are both in power-down states. In its STANDBY state, only the communication buffer is active in order to maintain information of the input data filled in by the buffer.

There are two types of transmission methods: (1) constant active mode (CAM), and (2) power saving mode (PSM). In the CAM, a node remains in an IDLE state whenever it is waiting for a data packet, and consumes a significant amount of energy due to high energy consumption in the/its IDLE state. Fig. 5 shows the operation modes of CAM.

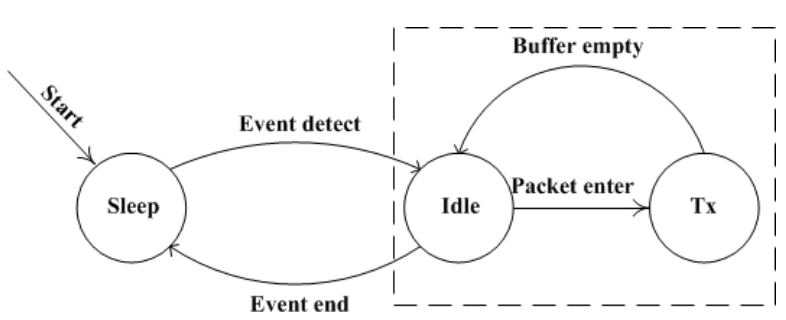

(a)

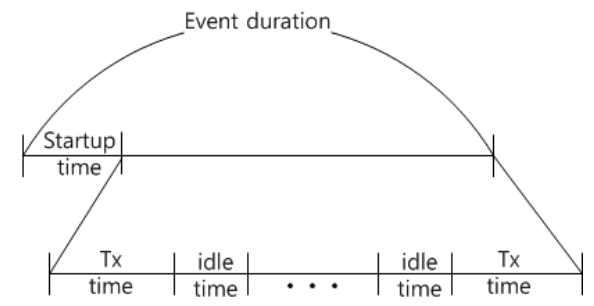

(b)

Fig. 5. Overall CAM operation, (a) State diagram of CAM, (b) Time diagram of CAM

In the PSM, a node changes its state to STANDBY when it waits for a transmission buffer to be filled. Fig. 6 (a) depicts the state transition of PSM. Fig. 6 (b) shows the sequences of the/a PSM data transmission operation for an event. For each event, the data is divided into 
several chunks by the buffer size, as depicted by number in Fig. 6 (b). In addition, s the buffer is filled with incoming data, the radio is turned on (in PSM, the/a state transition from STANDBY to Tx occurs) to send the data. With each STANDBY to Tx transition, start-up overhead is incurred. Therefore, a node with PSM consumes significantly less energy than a node with CAM, while incurring start-up overhead energy and a time delay.

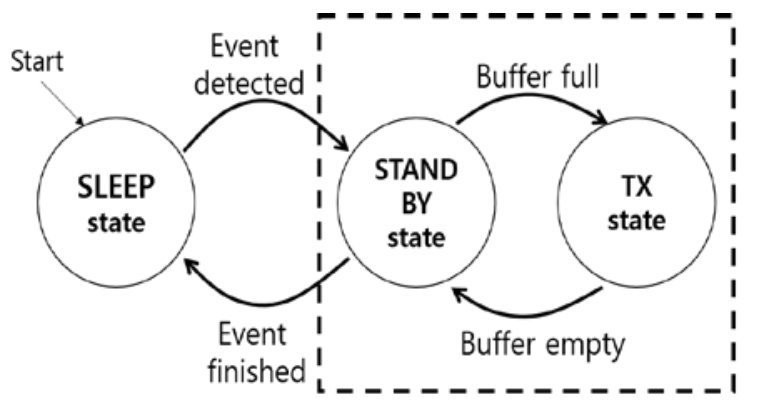

(a)

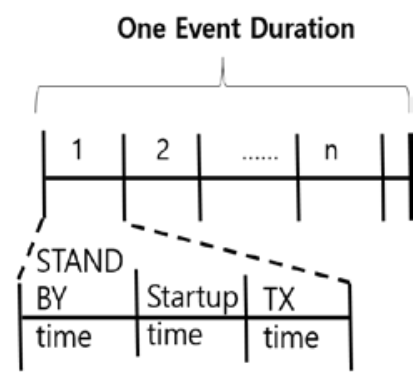

(b)

Fig. 6. Overall PSM operation , (a) State diagram of PSM, (b) Time diagram of PSM

We are here concerned with the key aspects of the transceiver part for focusing our strategy, which reduces communication energy. As the memory size increases, SRAM memory power increases. Fig. 7 shows the power consumption of $65 \mathrm{~nm}$ technology SRAM of different sizes, computed using a CACTI [10] tool. Note that as memory capacity increases, both leakage power and dynamic power seem to increase proportionally.

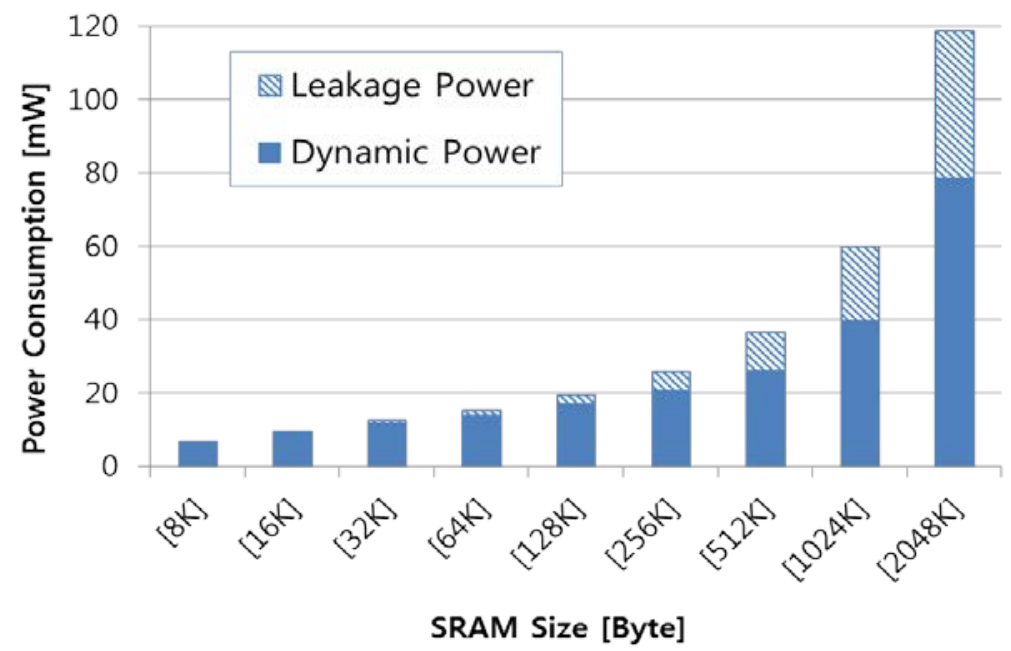

Fig. 7. SRAM leakage and dynamic power consumption

Fig. 8 shows the transceiver energy consumption as the communication block works in CAM and PSM for events of varying/different duration. As shown in Fig. 8, Wi-Fi with PSM consumes less energy than Wi-Fi with CAM. 


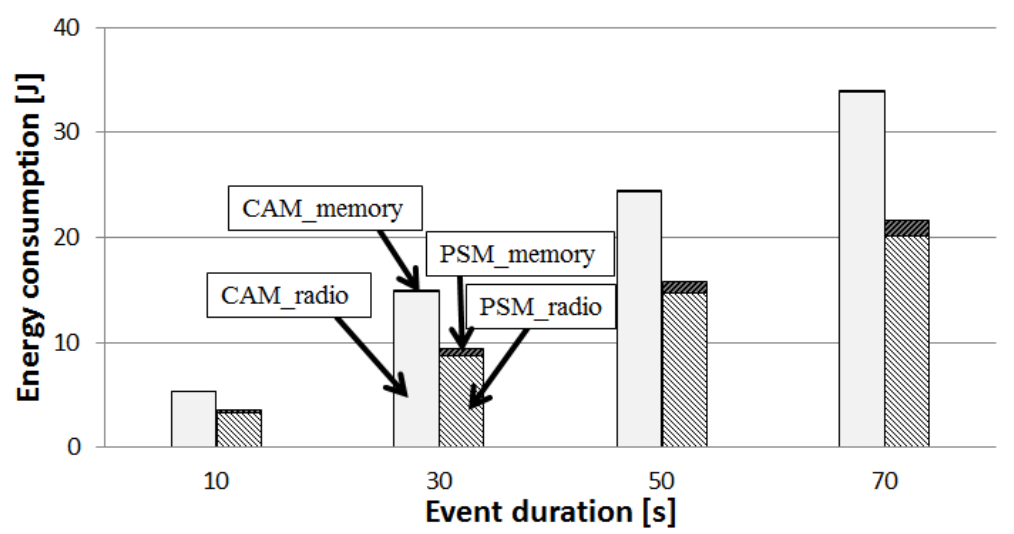

Fig. 8. Energy consumption by Wi-Fi anf Buffer in CAM and PSM (image size $=\mathrm{CIF}$, frame rate $=30 \mathrm{fps}$, and compression $=0.6 \mathrm{bpp}$ )

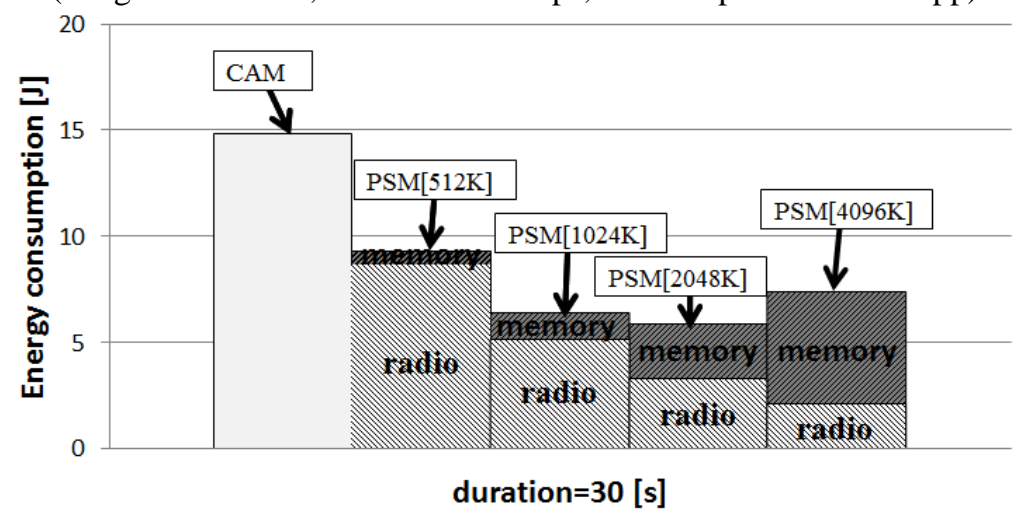

Fig. 9. Energy consumption of CAM and PSM for the different buffer size (image size $=\mathrm{CIF}$, frame rate $=30 \mathrm{fps}$, and compression $=0.6 \mathrm{bpp})$

Note that there is an optimal buffer size, which is 2048KB, as noted in Fig. 9. Our goal is to find the optimal buffer size for any given condition. We briefly explain the reasons below.

The Wi-Fi consumes a significant amount of power, even in the IDLE state, and there is energy overhead for a mode transition from a SLEEP to a Tx state. Therefore, to save Wi-Fi energy consumption, the radio needs to be turned off while the buffer is receiving data from the camera. However, frequent On/Off transitions may result in more energy consumption because this process incurs energy overhead. To reduce the On/Off transition frequency and power consumption of a radio, the buffer size needs to be as large as possible. However, a large-sized SRAM buffer causes not only excessive latency, but also significant additional power consumption, as shown in Fig. 9.

\section{Problem Definition and Formulation}

The input vatiables to our problem consist of four parts: Wi-Fi radio characteristics (Table 1), SRAM energy consumption, encoded video data size per second $\left(D_{e n}\right)$, and wireless channel bandwidth $\left(B W_{c h}\right)$. Given the above variables, our problem is to find the optimal SRAM size $\left(N_{S R A M}^{o p t}\right)$ for the/a communication buffer with the objective being to minimize the overall transceiver energy consumption $\left(E_{t r}\right)$. 
The problem is formulated as follows.

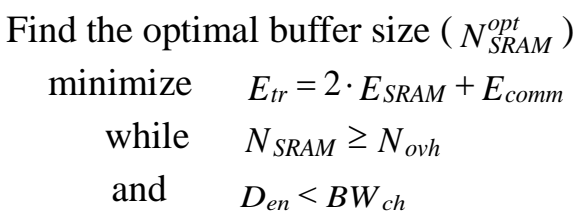

where $E_{t r}$ indicates a combination of both the receiver and sender transceiver energy consumption levels/factors/ratios, including the communication buffer and radio. $E_{S R A M}$ represents the energy consumption of the communication buffer, and $E_{\text {radio }}$ is radio energy, considering both the sender and receiver. Note that factor 2 in Eq. (1) reflects the energy consumption of the receiver-side buffer as well as the sender-side buffer. $N_{S R A M}$ is the buffer size and $\Delta N_{S R A M}$ is the size of video data generated from the encoder during radio turn-on delay as shown in Fig. 10.

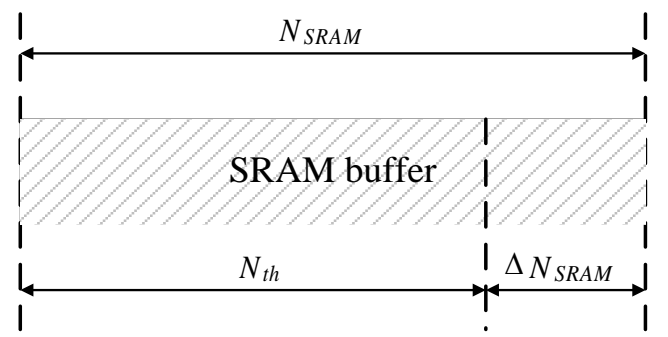

Fig. 10. When the SRAM communication buffer is full $\left(\mathrm{N}_{\mathrm{SRAM}}-\Delta \mathrm{N}_{\mathrm{SRAM}}\right)$, the radio is turned on to enable transmission.

The minimum buffer space during the start-up time $\left(\Delta N_{S R A M}\right)$ is expressed as $t_{\text {start }}^{\text {radio }} \cdot D_{\text {en }}$, where $D_{e n}$ represents width $_{\text {image }} \cdot$ height $_{\text {image }} \cdot$ frame_rate and $t_{\text {start }}^{\text {radio }}$ is the start-up time ratio, and $B W_{c h}$ is the channel bandwidth of the radio. The radio is powered on when the buffer is filled up to $N_{\text {SRAM }}-\Delta N_{\text {SRAM }}$, where nonzero $\Delta N_{\text {SRAM }}$ is needed to prevent the possible loss of data before the radio is fully on.

\subsection{Energy Consumption in Constant Active Mode (CAM)}

The Wi-Fi node in CAM is in an IDLE state when data transmission does not occur. When an event occurs, the transceiver initiates the TRANSMIT / RECEIVE state immediately, and repeatedly alternates between the IDLE and TRANSMIT / RECEIVE states. Depending on the relation between encoded data size per second $\left(D_{e n}\right)$ and channel bandwidth of the radio $\left(B W_{c h}\right)$, the energy consumption of the communication buffer $\left(E_{S R A M}\right)$ and the communication energy for both the sender and receiver nodes $\left(E_{\text {СомM }}\right)$ can be expressed as two different contexts. The formulations of the two different situations are given below.

If $D_{e n}$ is less than $B W_{c h}$, the energy consumption of the communication buffer $\left(E_{S R A M}\right)$ is expressed as follows. 


$$
E_{S R A M}=E_{\text {dyn }}^{S R A M}+E_{\text {leak }}^{S R A M}=2 \cdot e_{\text {dyn }}^{\text {SRAM }} \cdot D_{\text {en }} \cdot t_{e}+P_{\text {leak }}^{S R A M} \cdot t_{e}
$$

,where $e_{d y n}^{\text {SRAM }}$ denotes SRAM read/write dynamic energy per byte, $P_{\text {leak }}^{\text {SRAM }}$ is SRAM leakage power, and $t_{e}$ is an event duration time. Communication energy for both sender and receiver nodes $\left(E_{\text {comm }}\right)$ is expressed as follows.

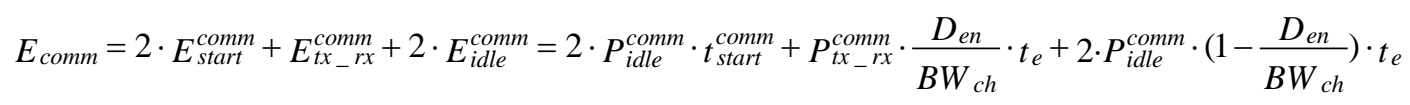

,where $E_{t x_{r} r x}^{c o m m}$ represents the energy consumed by transmitting and receiving data packets, $E_{\text {idle }}^{\text {comm }}$ is the idle state energy consumption, and $E_{\text {start }}^{\text {comm }}$ is the energy overhead due to the radio start-up process. $P_{\text {idle }}^{\text {comm }}, P_{t x_{-} r x}^{\text {comm }}$ represents power consumed in the idle, transmitting/receiving states, respectively. $t_{\text {start }}^{\text {comm }}$ is the start-up time.

If $D_{e n}$ is larger than $B W_{c h}$, the energy consumption of the communication buffer $\left(E_{S R A M}\right)$ is expressed as follows.

$$
E_{S R A M}=E_{d y n}^{S R A M}+E_{\text {leak }}^{S R A M}=2 \cdot e_{\text {dyn }}^{S R A M} \cdot D_{e n} \cdot t_{e}+P_{\text {leak }}^{S R A M} \cdot \frac{D_{\text {en }}}{B W_{c h}} \cdot t_{e}
$$

Communication energy for both the sender and receiver nodes $\left(E_{\text {comm }}\right)$ is expressed as follows.

$$
E_{\text {comm }}=2 \cdot E_{\text {start }}^{\text {comm }}+E_{t x_{-} r x}^{\text {comm }}=2 \cdot P_{\text {idle }}^{\text {comm }} \cdot t_{\text {start }}^{\text {comm }}+P_{t x_{-} r x}^{\text {comm }} \cdot \frac{D_{e n}}{B W_{c h}} \cdot t_{e}
$$

\subsection{Energy Consumption in the Power Saving Mode (PSM)}

In regard to the PSM, which is a more energy-efficient operation mode than CAM, the energy consumption of the communication buffer $\left(E_{S R A M}\right)$ is expressed as follows.

$$
\begin{aligned}
& E_{S R A M}=E_{\text {dyn }}^{S R A M}+E_{\text {leak }}^{S R A M} \\
& =2 \cdot e_{\text {dyn }}^{S R A M} \cdot D_{\text {en }} \cdot t_{e}+P_{\text {leak }}^{S R A M} \cdot\left(t_{e}+t_{\text {start }}^{\text {radio }}+\frac{N_{\text {res }}}{B W_{\text {ch }}}\right)
\end{aligned}
$$

where $e_{d y n}^{S R A M}$ denotes SRAM read/write dynamic energy per byte, $P_{\text {leak }}^{S R A M}$ is SRAM leakage power, $N_{\text {res }}$ is the amount of the residual data to send last, $t_{e}$ is an/the event duration time, and $t_{\text {start }}^{\text {radio }}$ is the radio start-up time (turn-on delay). Radio energy, considering both the sender and receiver nodes $\left(E_{\text {radio }}\right)$, is expressed as follows. 


$$
\begin{gathered}
E_{\text {radio }}=n \cdot E_{f b u f}^{\text {radio }}+E_{\text {res }}^{\text {radio }} \\
=n \cdot\left(2 \cdot E_{\text {start }}^{\text {radio }}+E_{t x_{-} r x}^{f b u f}\right)+2 \cdot E_{\text {start }}^{\text {radio }}+E_{t x_{-} r x}^{\text {res }} r x
\end{gathered}
$$

where $E_{f b u f}^{\text {radio }}$ and $E_{\text {res }}^{\text {radio }}$ denote radio energy required to send each data set/sequence filled in the buffer and the final residual data in the buffer, respectively. The encoded video data is divided by n chunks according to the SRAM buffer size, as shown in Eq. (10). In addition, $n$ also represents the number of mode switches from STANDBY to Tx/Rx. In Eq. (9), $E_{t x_{-} r x}^{f b u f}$ and $E_{t x_{-} r x}^{r e s}$ are the energy rates/levels/ratios consumed by both transmitting and receiving data packets in the full-buffer and residual-buffer state, respectively, as shown in Eq. (12) and (9). $E_{\text {start }}^{\text {radio }}$ is the radio start-up energy overhead required/needed to turn on the radio(s), which

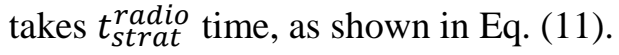

$$
\begin{aligned}
& n=\left\lfloor\frac{D_{e n} \cdot t_{e}}{B W_{c h} \cdot t_{o p}^{r a d i o}}\right\rfloor \\
& E_{\text {start }}^{\text {radio }}=P_{\text {idle }}^{\text {radio }} \cdot t_{\text {start }}^{\text {radio }} \\
& E_{t x_{-}{ }_{r X}}^{f b u f_{t}}=P_{t x_{-} r x_{1}}^{\text {radio }} \cdot t_{o p}^{\text {radio }} \\
& E_{t x_{-} r x}^{r e s}=P_{t x_{-} r x}^{r a d i o} \cdot \frac{N_{r e s}}{B W_{c h}} \\
& t_{o p}^{\text {radio }}=\frac{N_{S R A M}}{B W_{c h}-D_{e n}} \\
& N_{\text {res }}=D_{e n} \cdot t_{e}-n \cdot\left(B W_{c h} \cdot t_{o p}^{\text {radio }}\right)
\end{aligned}
$$

In Eq. (11) and (12), $P_{i d l e}^{\text {radio }}$ and $P_{t x_{-} r x}^{\text {radio }}$ represents he radio power consumed in the IDLE and $\mathrm{Tx} / \mathrm{Rx}$ states, respectively. In Eq. (14), $t_{o p}^{\text {radio }}$ is the radio operation time per full-buffer state. To eliminate the flooring operator $(\lfloor\cdot\rfloor)$,Eq. (10) can be rewritten as Eq. (16), which also allows the equation differentiable.

$$
\mathrm{n}=\left\lfloor\frac{D_{e n} \cdot t_{e}}{B_{c h} \cdot t_{o p}}\right\rfloor=\left(\frac{D_{e n} \cdot t_{e}}{B_{c h} \cdot t_{o p}}\right)-\alpha, \quad(0 \leq \alpha<1)
$$

By the Eq. (11) to (16), Eq. (9) can be expressed as Eq. (17).

$$
E_{\text {radio }}=\frac{2 \cdot D_{e n} \cdot t_{e} \cdot\left(B W_{c h}-D_{e n}\right) \cdot E_{\text {start }}^{\text {radio }}}{B W_{c h} \cdot N_{S R A M}}+\frac{D_{e n} \cdot t_{e} \cdot P_{t x \_r x}}{B W_{c h}}
$$




\section{Optimal Buffer Size Selection}

\subsection{Solving Optimal Buffer Size Formula}

In this section, we explain how to determine the optimal buffer size $\left(N_{S R A M}^{o p t}\right)$ regarding energy consumption minimization in the power saving mode(PSM). We approximate the SRAM dynamic energy per byte $\left(e_{d y n}^{S R A M}\right)$ and leakage power consumption $\left(P_{\text {leak }}^{\text {SRAM }}\right)$, as shown in Eq. (18) and (19), respectively. We assume that the dynamic energy and leakage energy (power) are all proportional to the layout area and, therefore, can be represented as a sum of two components, a term proportional to $N_{S R A M}$ and a constant term. Models in Eq. (18) and (19) are fitted with Matlab to approximate the SRAM power consumption data obtained by CACTI [10]. The constants $p_{1}, p_{2}, q_{1}$, and $q_{2}$ in Eq. (18) and (19) are the fitting constants. ${ }^{2}$

$$
\begin{array}{r}
e_{\text {dyn }}^{\text {SRAM }}=p_{1} \cdot N_{\text {SRAM }}+p_{2} \\
P_{\text {leak }}^{\text {SRAM }}=q_{1} \cdot N_{S R A M}+q_{2}
\end{array}
$$

The parameters in Eq. (18) and Eq. (19) are fitted to model SRAM power consumption data obtained by CACTI [10], resulting in $\mathrm{p}_{1}=2.15 \times 10^{-17}, \mathrm{p}_{2}=2.06 \times 10^{-12}$ and $\mathrm{q}_{1}=1.93 \times 10^{-08}$, $\mathrm{q}_{2}=0.00011$. By substituting $P_{\text {leak }}^{S R A M}$ and $e_{d y n}^{S R A M}$ in Eq. (8) with Eq. (18) and (19), we can express the $E_{S R A M}$ as a function of $N_{S R A M}$, as illustrated in Eq. (20).

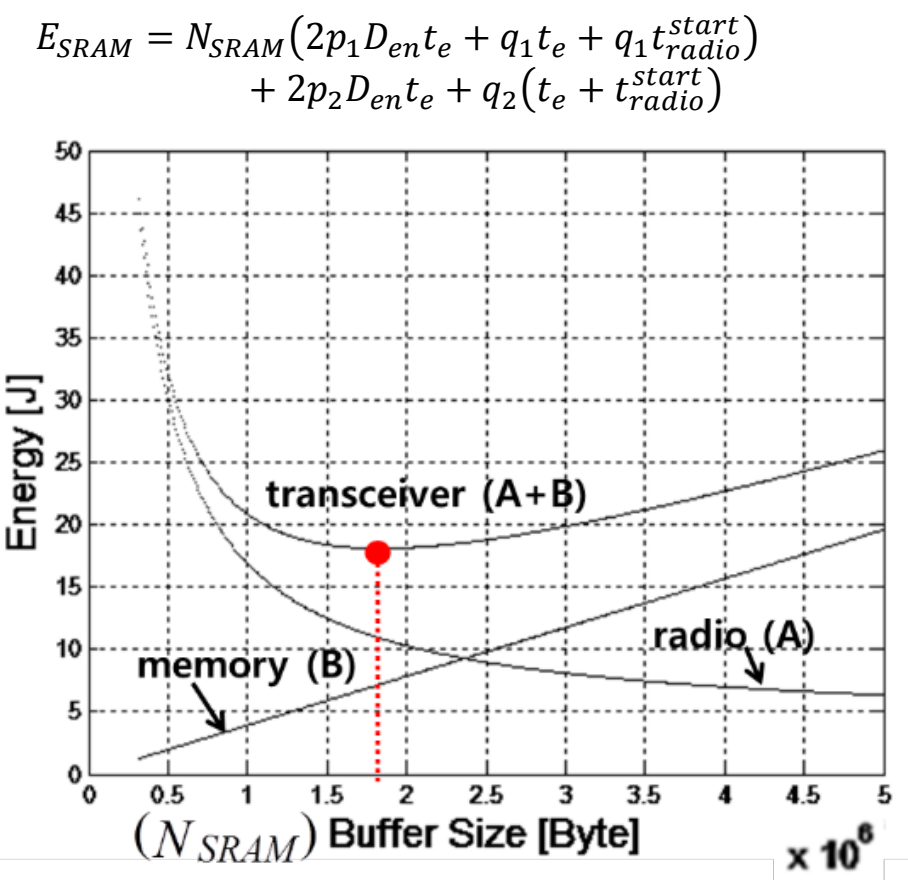

Fig. 11. Optimal Buffer size selection (red point) is such that the total energy consumption (memory + radio energy) is minimal (CIF, 30fps, $0.6 \mathrm{bpp}$ ): calculated without round-off operation.

\footnotetext{
${ }^{2}$ In our experiments, $\mathrm{p}_{1}=2.15 \mathrm{E}-17, \mathrm{p}_{2}=2.06 \mathrm{E}-12, \mathrm{q}_{1}=1.93 \mathrm{E}-08, \mathrm{q}_{2}=0.0011$.
} 
Fig. 11 plots Eq. (13), Eq. (16), and the sum of them as $E_{t r}$. In Fig. 11, as the $N_{S R A M}$ increases, the energy consumption of radio $\left(E_{\text {radio }}\right)$ monotonically decreases while the energy consumption of the buffer $\left(E_{S R A M}\right)$ linearly increases. Due to the trade-off relationship between $E_{\text {radio }}$ and $E_{S R A M}$, there is an optimal point $\left(N_{S R A M}^{\text {opt }}\right)$ where $E_{t r}$ is minimized, which is marked as a dot in Fig. 11. The graph of $E_{t r}$ represents a convexity with respect to $N_{S R A M}$.

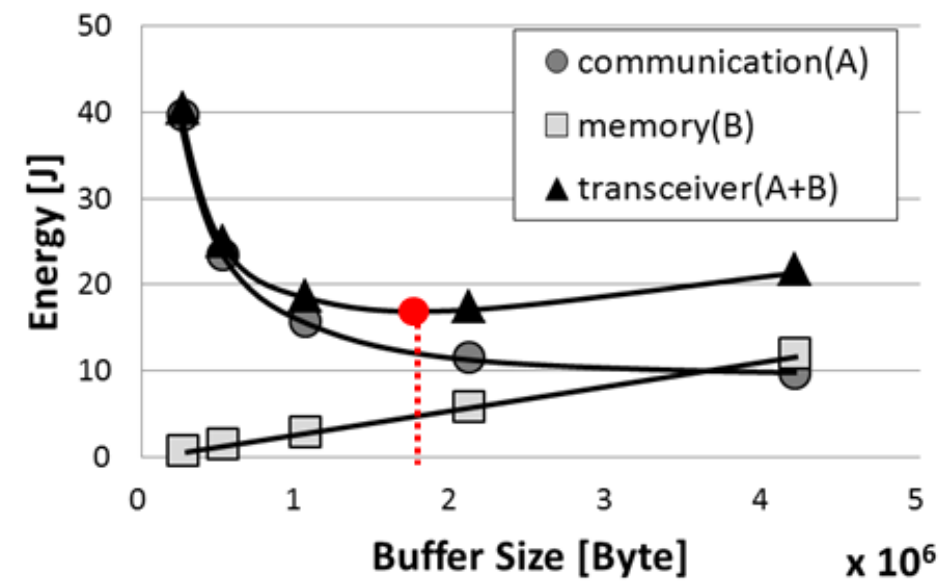

Fig. 12. Optimal Buffer size selection (red point) is such that the total energy consumption (memory + radio energy) is minimal (CIF, 30fps, 0.6bpp) : calculated by rounding-off operation.

Fig. 12 shows the $E_{t r}$ has convexity with respect to $N_{S R A M}$. In order to make $E_{t r}$ differentiable, with respect to size $N_{S R A M}$, we omitted the floor operator(l. J).

Now, we can calculate $N_{S R A M}^{o p t}$ by finding the point that satisfies $\partial E_{t r} / \partial N_{S R A M}=0$ as in Eq. (17).

$$
N_{S R A M}^{\text {opt }} \Rightarrow \frac{\partial E_{t r}}{\partial N_{S R A M}}=\frac{\partial\left(2 \cdot E_{S R A M}+E_{\text {radio }}\right)}{\partial N_{S R A M}}=0
$$

As a result, the solution of Eq. (17) is shown in Eq. (18) ${ }^{3}$.

$$
N_{S R A M}^{\text {opt }}=\sqrt{\frac{D_{e n} \cdot\left(B W_{c h}-D_{e n}\right) \cdot 2 \cdot P_{i d l e}^{\text {radio }} \cdot t_{\text {start }}^{\text {radio }}}{B W_{c h} \cdot\left(4 \cdot p_{1} \cdot D_{e n}+2 \cdot q_{1}\right)}}
$$

By Eq. (18), we can see that $N_{S R A M}^{o p t}$ is a function of encoded video data size per second $\left(D_{e n}\right)$, and channel bandwidth is/represents $\left(B W_{c h}\right)$. Fig. 13 llustrates the optimal buffer size for $D_{e n}$ and $B W_{c h}$. In Fig. 13, $N_{S R A M}^{o p t}$ increases as $D_{e n}$ or $B W_{c h}$ increases, while it decreases as $D_{e n}$ further increases, as calculated by BWch=16Mbps. Based on the above discussion, we can conclude that optimal buffer sizes vary according to Den and $B W_{c h}$.

\footnotetext{
${ }^{3}$ We assumed that $\alpha=0$ in Eq (12) and $t_{e} \gg t_{\text {radio }}^{\text {start }}$ in Eq (4)
} 


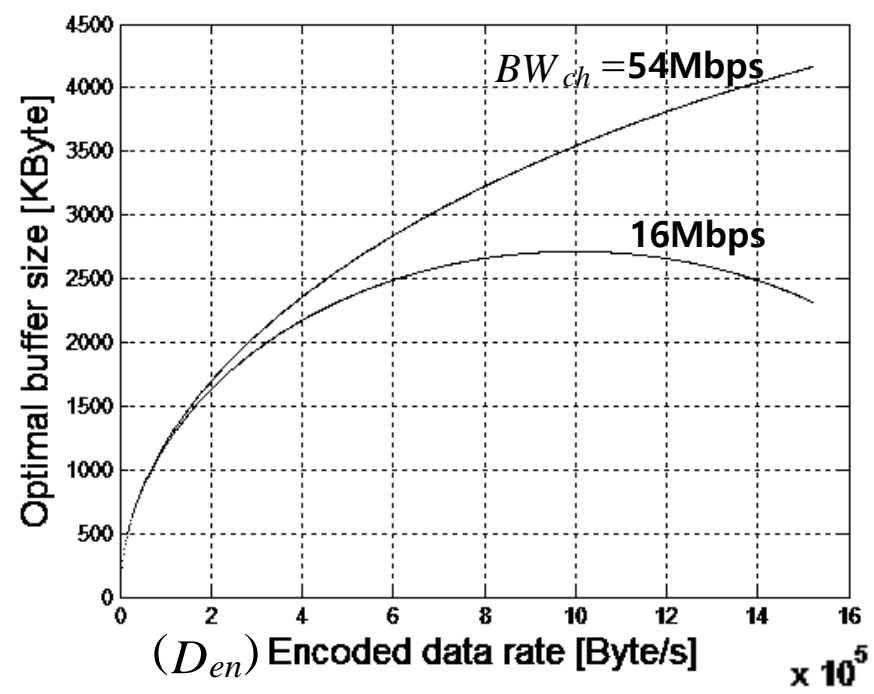

Fig. 13. Optimal buffer size selection depends on the incoming encoded data rate ( $\left.D_{\text {en }}\right)$ and channel bandwidth ( $B W_{c h}$ ) as the two curves showtransmission rates of 54Mbps and $16 \mathrm{Mbps}$, respectively.

\subsection{Revised System Design for Practical Application}

It is necessary to select the optimal buffer size dynamically by using power-gated SRAM buffer memory. Power-gating is a common technology purposed to save energy by shuttingdown the power supply of individual circuit parts in SoC design environments[11,13]. By means of the run-time PG(power gating) technique, the effective buffer size can be changed in a dynamic manner. Fig. 14 shows the optimal buffer size for each video resolution and frame rates in order to minimal WVSN energy consumption.

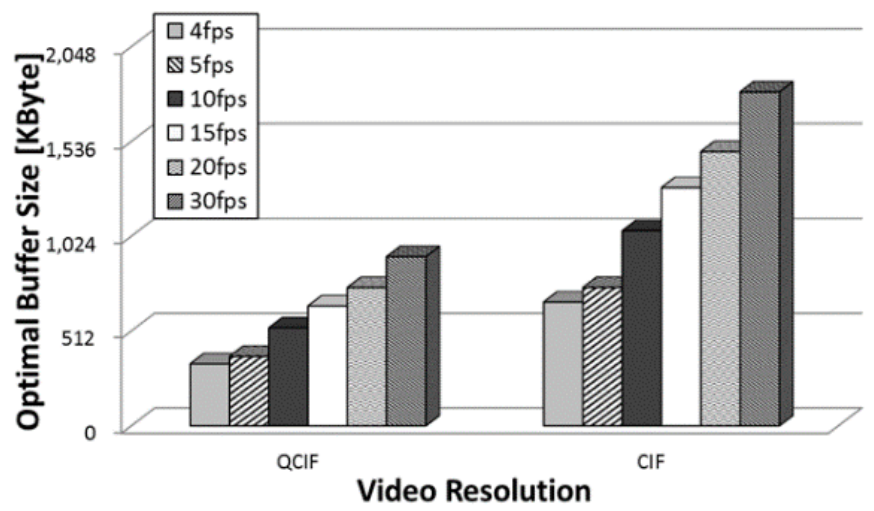

Fig. 14. The energy efficient buffer size for different video resolution and frame rates.

Our WVSN nodes periodically detect $D_{e n}$ and $B W_{c h}$, then the SRAM buffer size is dynamically adjusted by the buffer resizing method coupled with a PG technique. Our buffer resizing algorithm affects not only the energy consumption of the buffer itself, but also that of the Wi-Fi radio, including the power amplifier. Fig. 15 is a revised version of the control flow as illustrated in Fig. 4. Fig. 15 is a refined system control flow that changes the buffer size adaptively by means of power-gating technology. 


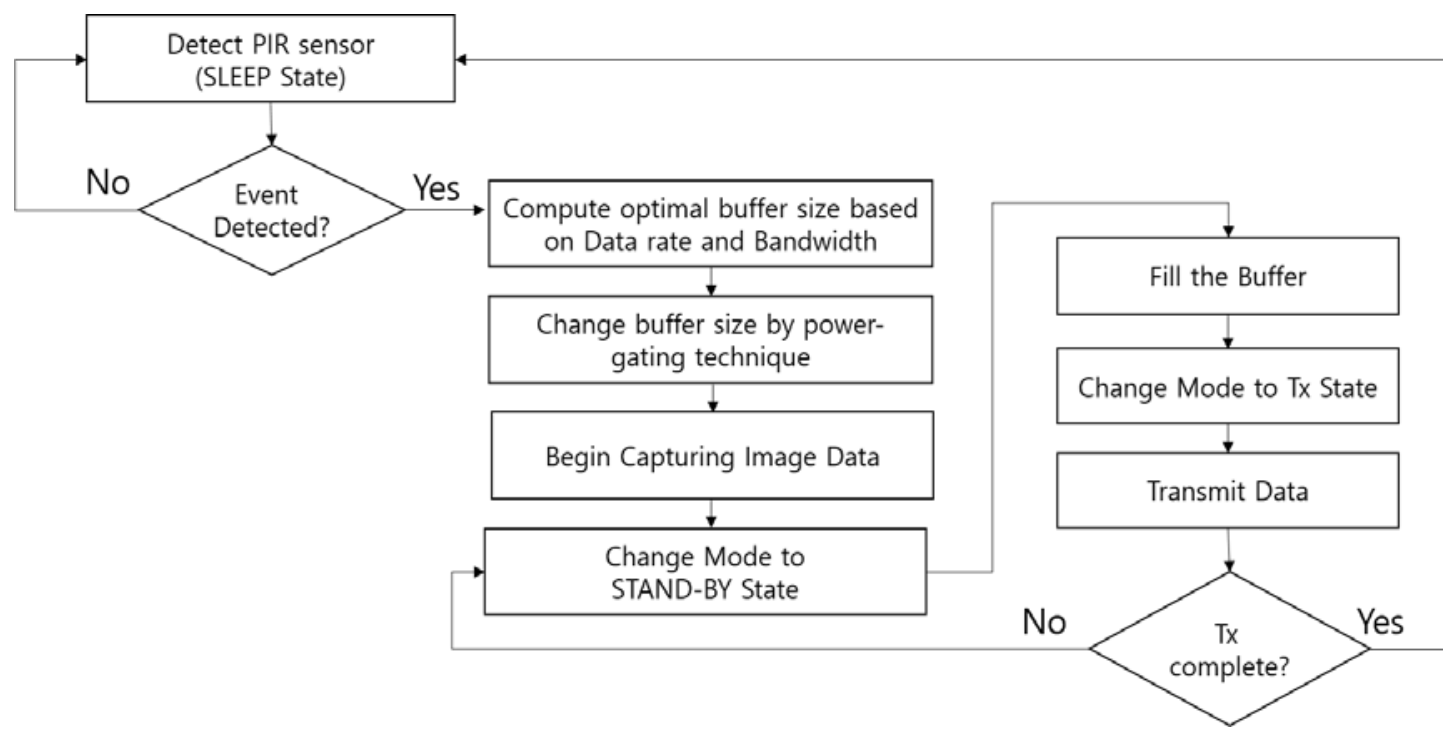

Fig. 15. The revised flow chart for the/a WVSN node with dynamic buffer resizing

\section{Experimental Results}

In the experiments, we used simulations to measure the power consumption data in WVSN nodes with real scenarios. We built an experimental setup, as shown in Table 2. Within this setting, we performed experiments using five sample video sequences of campus surveillance video with different occurrence rates, ranging from $1.74 \%$ to $10.81 \%$.

We also altered the channel bandwidth to reflect wireless channel condition variations in the experiments. We adjusted the buffer size dynamically according to the sampling data rate and channel bandwidth changes.

Table 2. Experimental Sttup

\begin{tabular}{|c|c|}
\hline $\begin{array}{c}\text { Captured Image Resolution by } \\
\text { CIS }\end{array}$ & CIF (352 x 288) @ 30fps [14] \\
\hline Event detector & PIR sensor [15] \\
\hline Video encoder & 0.6 bpp(bits per pixel) by \\
& H.264 with baseline profile \\
\hline SRAM transceiver buffer & CACTI with $65 \mathrm{~nm}$, \\
& low power process [10] \\
\hline Wi-Fi transceiver & Same as Table 1 \\
\hline Battery & $3600 \mathrm{mWh}$ (alkaline AA battery) \\
\hline
\end{tabular}

Fig. 16 shows the energy consumption of the radio and SRAM buffer for different sampling rates obtained from the experiments. As shown in this figure, we compare the energy consumption of the CAM and PSM transceivers with a fixed buffer size (512Kbyte) [12], and that of the PSM transceiver with the/an optimal buffer sizing method (hereafter, Opt_PSM). Fig. 16 (a) shows the results based on deal radio performance ( $\left.B W_{c h}=54 \mathrm{Mbps}\right)$, and Fig. 16 (b) shows the results with practical radio performance in a congested traffic situation $\left(B W_{c h}=16 \mathrm{Mbps}\right)$. In either case, the Opt_PSM shows the best results. In regards to the ideal data rate $\left(B W_{c h}=54 \mathrm{Mbps}\right)$, the Opt_PSM reduced $E_{t r}$ from $13.8 \%$ to $40.48 \%$ compared to the PSM with a/the fixed buffer size (512KB)[12]. In the case of the practical data rate $\left(B W_{c h}\right.$ 
$=16 \mathrm{Mbps})$, the Opt_PSM reduced $E_{t r}$ from $12.01 \%$ to $29.79 \%$ compared to the PSM with a/the fixed buffer size [12].

Fig. 17 shows the normalized transceiver energy consumption $\left(E_{t r}\right)$. In regards to ideal data rate, Wi-Fi(Opt_PSM) reduced $E_{\text {tr }}$ from $13.8 \%$ to $40.48 \%$ compared to the PSM with a/the fixed buffer size (512KB) [12]. In Addition, the effective data rate, the Wi-Fi(Opt_PSM) reduced $E_{t r}$ from $12.01 \%$ to $29.79 \%$ compared to the method [12]. Note that the energy consumption of memory part of OPT_PSM is increased than that of CAM and PSM. But, the energy consumption of radio part of OPT_PSM has much reduced than that of CAM and PSM, resulting in overall energy saving with OPT_PSM approach.

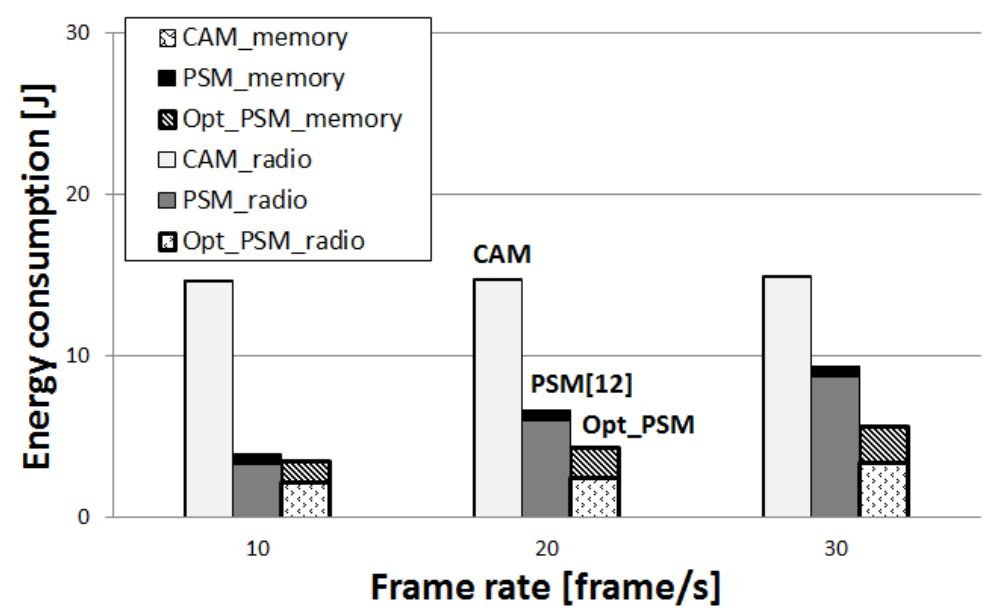

(a)

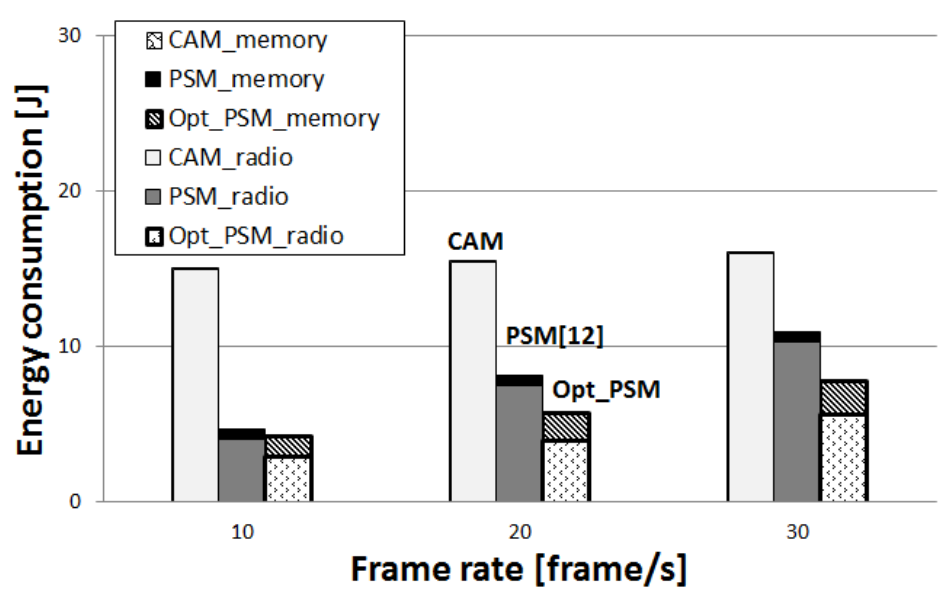

(b)

Fig. 16. Energy consumption of the transceiver $\left(E_{t r}\right)$ for an event for three different approaches, CAM, PSM, Opt_PSM for CIF image size with three different frame rates. (a) For ideal data rate ( $\left.B W_{c h}=54 \mathrm{Mbps}\right)$, Optimal buffer size : 1053Kbyte(10fps), 1481 Kbyte (20fps), 1803 Kbyte(30fps), (b) For a practical data rate $\left(B W_{c h}=16 \mathrm{Mbps}\right)$, Optimal buffer size : $1039 \mathrm{Kbyte}(10 \mathrm{fps}), 1439$ Kbyte (20fps), 1727 Kbyte(30fps). 


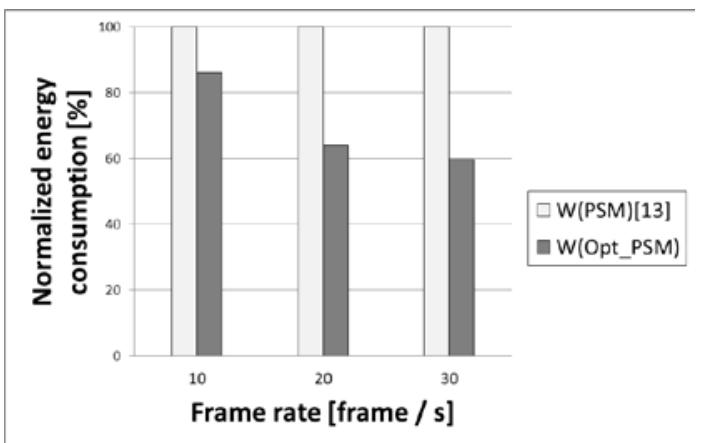

(a)

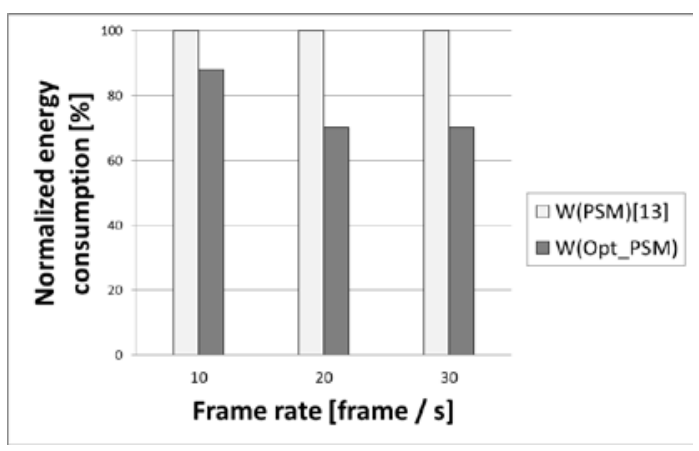

(b)

Fig. 17. Normalized $E_{\text {sys }}$ (transceiver part) energy consumption for an event with different approaches, (a) ideal data rate, (b) effective data rate

We also showed the lifetime of WVSN node with randomly changing event duration, frame rate, and channel condition in Fig. 18, which shows that our method prolongs the system lifetime from $20.46 \%$ to $24.6 \%$ compared to the PSM with fixed buffer size [12].

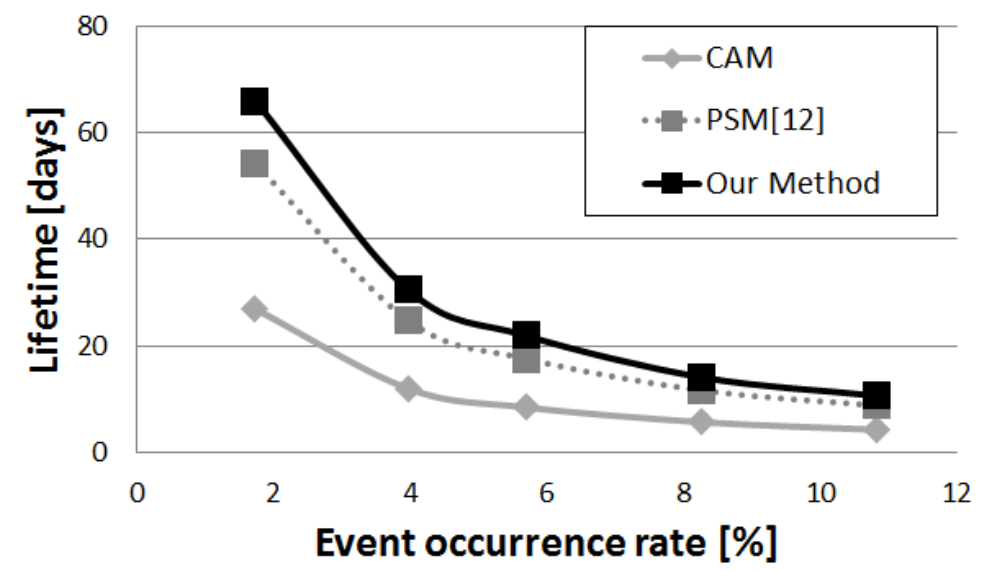

Fig. 18. Lifetime of a camera node with $1 \mathrm{AA}$ alkaline battery, where the frame rate is varied as 10fps, 20fps, 30fps randomly, and channel condition is varied between the ideal data rate $\left(B W_{c h}=54 \mathrm{Mbps}\right)$ and practical data rate $\left(B W_{c h}=16 \mathrm{Mbps}\right)$ randomly.

\section{Conclusion}

In this paper, we proposed an energy-aware dynamic communication buffer resizing of nodes in wireless visual sensor networks. Buffer size is critical, as it affects not only the energy consumption of the buffer itselWof, but also that of the Wi-Fi radio. This includes the power amplifier, as significant power consumption during the ON/OFF switching is affected by the frequency of buffer emptying, which is, in turn, determined by the buffer size. The proposed algorithm for determining the optimal buffer size is based on the analytic formula for communication energy.

Our buffer resizing approach can be utilized in the WVSN node design through coupling with a dynamic SRAM buffer power-gating technique. Experimental results with real video sequences show that the energy reduction of communication parts by our approach is up to $40.48 \%$ (26.96\% on average) compared to the conventional communication scheme with a/the 
fixed buffer size. Based on the simulation results, our approach is expected to extend the lifetime of wireless camera nodes by $22.17 \%$ on average compared to conventional communication approaches.

\section{References}

[1] S. Soro and W. Heinzelman, "A survey of visual sensor networks." Advances in Multimedia, pp. 1-21, 2009. Article(CrossRefLink)

[2] H. Lakdawala, et al., "32nm x86 OS-compliant PC on-chip with dual-core Atom ${ }^{\circledR}$ processor and RF WiFi transceiver," in Proc. of IEEE Int'l Solid-State Circuits Conf. (ISSCC), pp.62-64, Feb. 2012. Article(CrossRefLink)

[3] S. Na, et al., "Lifetime maximization of video blackbox surveillance camera," in Proc. of IEEE Conf. on Multimedia and Expo (ICME), pp.1-6, July 2011. Article(CrossRefLink)

[4] A. Zainaldin, et al., "Adaptive Rate Control Low bit-rate Video Transmission over Wireless ZigBee networks," in Proc. of IEEE International Conference on Communications, pp. 52-58, May, 2008. Article(CrossRefLink)

[5] Schurgers, et al., "Energy efficient routing in wireless sensor networks," MILCOM 2001 Communications for Network-Centric Operations: Creating the Information Force, Oct. 2001. Article(CrossRefLink)

[6] Krashinsky and Balakrishnan, "Minimizing energy for wireless web access with bounded slowdown," in Proc. of the 8th ACM annual international conference on Mobile computing and networking, pp. 119-130, 2002. Article(CrossRefLink)

[7] T. Enhua, et al., "PSM-throttling: Minimizing Energy Consumption for Bulk Data Communications in WLANs," IEEE International Conference on Network Protocols, ICNP 2007, pp.123-132, Oct. 2007. Article(CrossRefLink)

[8] Lee, Y., Kim, J., Kyung, C.-M., "Energy-Aware Video Encoding for Image Quality Improvement in Battery-Operated Surveillance Camera," IEEE Trans. on VSLI Systems, vol. 20, no. 2, pp. 310-318, 2012.Article(CrossRefLink)

[9] S. Drago, et al., "A 2.4GHz 830pJ/bit duty-cycled wake-up receiver with $-82 \mathrm{dBm}$ sensitivity for crys-tal-less wireless sensor nodes," in Proc. Of IEEE Int'l Solid-State Circuits Conference (ISSCC), pp.224-225, Feb. 2010. Article(CrossRefLink)

[10] CACTI http://quid.hpl.hp.com:9081/cacti/sram.y?new.

[11] Kyung, C.M., Yoo, S., Energy-Aware System Design, (Springer, 2011, 1st ed.) Article(CrossRefLink)

[12] Yong He, Ruixi Yuan, "A Novel Scheduled Power Saving Mechanism for 802.11 Wireless LANs," IEEE Trans. on Mobile Computing, vol. 8, no. 6, pp. 1368-1383, Oct. 2009. Article(CrossRefLink)

[13] Kang, K., Kim, J., Yoo,S., Kyung, C.M., "Temperature-Aware Integrated DVFS and Power gating for Executing tasks with Runtime Distribution,” IEEE Trans. on CAD, vol. 29, no. 9, pp. 1381-1394, 2010. Article(CrossRefLink)

[14] J. Choi, et al., "A 1.36 $\mathrm{W}$ adaptive CMOS image sensor with reconfigurable modes of operation from available energy/illumination for distributed wireless sensor network," in Proc. of IEEE Int'l Solid-State Circuits Conf. (ISSCC), pp.112-114, Feb. 2012. Article(CrossRefLink)

[15] C. Tsai, et al., "PIR-sensor-based lighting device with ultra-low standby power consumption," in Proc . of IEEE In-strumentation and Measurement Technology Conf., (I2MTC), pp.1-6, May 2011. Article(CrossRefLink)

[16] T. Winkler, B. Rinner "Power aware communication in wireless pervasive smart camera networks," in Proc. of Int'l Conf. on Intelligent Sensors, Sensor Networks and Information Processing (ISSNIP), pp.283-288, Dec. 2009. Article(CrossRefLink) 


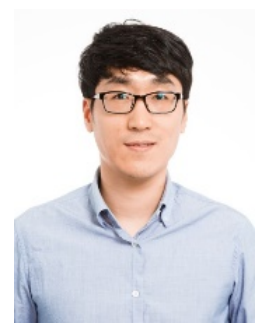

Kangwoo Choi received the B.S. degree in Electrical and Computer Engineering from University of Seoul in 2011, the M.S. degree in Electrical Engineering from KAIST in 2013. Since 2013, he works at Samsung Electronics Co., Ltd. where he is responsible for the development of cache coherent interconnect network. He has participated in many research topics including communication networks, multimedia codec, VLSI interconnects.

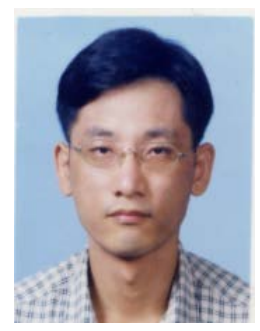

Kang Yi received his BS, MS, and Ph.D. degrees in computer engineering from Seoul National University, Korea in 1990, 1992 and 1997 respectively. The title of his thesis is a Unified Approach to Technology Mapping for FPGA. He worked for the Research Institute of Advanced Computer Technology, Seoul, Korea from September 1997 to February 1998. He was a full time Lecturer of department of Information and Communication Engineering in Inje University, Korea from March 1998 to February 1999. He joined Handong Global University in Feburary 1999 as full time lefturer in the School of Computer Science and Electrical Engineering, where he is now Professor. He was a visiting professor at University of Californioa, Irviine during 2005-2006 and was an inivited professor at Center for Integrated Smart Sensors (CISS) funded by Korean Government during 2013-2014. He has published and presented more than 100 papers and 10 books in the area of low power and low cost digital system design, multimedia computing, embedded systems, and reconfigurable computing. He also has more than 20 patents focusing on the area of computer vision, advanced drivers assistant systems and multimedia embedded systems. His current research interests covers System-on-a-chip design, reconfigurable computing, and image processing applications.

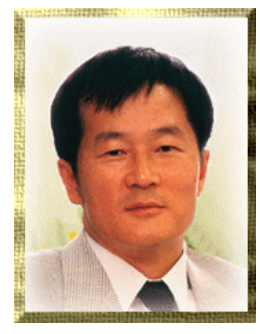

Chong-Min Kyung received the B.S. degree in Electronics Engineering from Seoul National University in 1975, the M.S. and Ph.D. degree in Electrical Engineering from KAIST in 1977 and 1981, respectively. From April 1981 to January 1983, he worked at Bell Telephone Laboratories, Murray Hill, New Jersey as a postdoc. He joined KAIST in February 1983 as Assistant Professor in the Department of Electrical Engineering, where he is now Professor. He founded IDEC (IC Design Education Center) in 1995 to support IC design education in Korean universities nationwide. He was a visiting professor at the Univ. Karsruhe, Germany, from in 1989, as an Alexander von Humboldt fellow, a visiting professor at the University of Tokyo in 1985, Chuo University in 2005, at the Technical Univ. of Munich during in 1994. at Waseda University during 2003-2006, at the University of Auckland, New Zealand during 2005-2006. His interested research area includes SoC(System-on-a-Chip) design and verification methodology, microprocessor and DSP architectures for low-power and various multi-media embedded applications, 3-D cameras and smart sensor systems.

He has published over 300 papers in the international conferences and journals in the SoC design, EDA algorithm, computer architecture and smart sensor systems. He received the Most Excellent Design Award, and Special Feature Award in the University Design Contest in the ASP-DAC 1997 and 1998, respectively. He received Best Paper/Design Awards in numerous international conferences including ASP-DAC 1997 and 1998, DAC in 2000, ICSPAT in 1999, ICCD in 1999, and ISQED in 2014. He is a member of National Academy of Engineering Korea, and Korean Academy of Science and Technology, and IEEE fellow. In 2011 he founded and currently leads a number of core projects including 3-D camera systems, air and water pollution monitoring systems, open-platform event logging systems as Director the Center for Integrated Smart Sensors (CISS), a Global Frontier Project supported for 9 years by Korean Government. 